\title{
A simplified quantum mechanical model of diatomic molecules
}

\author{
Nielsen, Lars Drud
}

Published in:

American Journal of Physics

Link to article, DOI:

10.1119/1.11510

Publication date:

1978

Document Version

Publisher's PDF, also known as Version of record

Link back to DTU Orbit

Citation (APA):

Nielsen, L. D. (1978). A simplified quantum mechanical model of diatomic molecules. American Journal of Physics, 46(9), 889-892. https://doi.org/10.1119/1.11510

\section{General rights}

Copyright and moral rights for the publications made accessible in the public portal are retained by the authors and/or other copyright owners and it is a condition of accessing publications that users recognise and abide by the legal requirements associated with these rights.

- Users may download and print one copy of any publication from the public portal for the purpose of private study or research.

- You may not further distribute the material or use it for any profit-making activity or commercial gain

- You may freely distribute the URL identifying the publication in the public portal

If you believe that this document breaches copyright please contact us providing details, and we will remove access to the work immediately and investigate your claim 


\title{
A simplified quantum mechanical model of diatomic molecules
}

\author{
Lars Drud Nielsen \\ Physics Laboratory III, Technical University of Denmark, DK-2800 Lyngby, Denmark \\ (Received 20 January 1977; accepted 20 April 1977)
}

\begin{abstract}
A one-dimensional molecule model with Coulomb potentials replaced by $\delta$ functions is introduced. The mathematical simplicity of the model facilitates the quantum mechanical treatment and offers a straightforward demonstration of the essentials of two-particle problems. In spite of the crudeness of the model, quantitative results are obtained in fair agreement with those of a true hydrogen molecule.
\end{abstract}

\section{INTRODUCTION}

Introductory courses in quantum mechanics very often have to refrain from dealing with more than one-particle problems. Two-particle problems, such as the covalent chemical bond in the hydrogen molecule, are not conceptually very difficult, but mere computational obstacles prevent the student from getting a quick understanding of the essentials in two-particle calculations. A variational approach to the ground-state solution of the $\mathrm{H}_{2}$ problem ${ }^{1}$ for instance involves computation of six-dimensional integrals in spheroidal coordinates, and a painstaking treatment serves mathematical training more than physical insight.

We have intended to suppress the mathematics and stress the physics by introducing a very crude model, characterized by the following two simplifications:

(i) The model is one dimensional, so that six-dimensional integrals are generally replaced by two-dimensional integrals.

(ii) Coulomb potentials, which are out of place in a onedimensional world anyhow, are replaced by $\delta$ functions. All integration procedures are thereby effectively reduced further by one dimension. Moreover, the natural trial functions turn out to be of very simple form (exponential functions).

Although the academic one-dimensional " $\delta_{2}$ molecule" seems far from resembling a real $\mathrm{H}_{2}$ molecule, the following calculations will demonstrate a close qualitative resemblance, and, as far as quantitative comparisons can be carried out, surprisingly fine agreements are found too.

\section{WAVE FUNCTIONS OF THE $\delta$ ATOM}

The time-independent Schrödinger equation for one electron in a one-dimensional potential of the form $V(x)$ $=-A \delta(x)(A>0)$ becomes

$$
-\left(\hbar^{2} / 2 m\right) v^{\prime \prime}(x)-A \delta(x) v(x)=E v(x) .
$$

which has the normalized solution

$$
\begin{gathered}
v(x)=\beta^{1 / 2} \exp (-\beta|x|), \\
\beta=m A / \hbar^{2}, \\
E=-\hbar^{2} \beta^{2} / 2 m=-\beta A / 2=-m A^{2} / 2 \hbar^{2} \equiv-E_{0} .
\end{gathered}
$$

This is the only bound solution that satisfies Eq. (1) also for $x=0 .^{2}$ The lack of excited states is a consequence of the short-range potential and is in extreme contrast to the hydrogen problem with its infinite number of excited states. However, this defect will not cause any troubles in the following considerations concerning only the molecular ground state. The positive constant $E_{0}$ will be the natural unit of energy, analogous to the Rydberg energy in the hydrogen case.

\section{$\delta_{2}$ MOLECULE}

Imagine a one-dimensional diatomic molecule with $\delta$ nuclei $a$ and $b$ fixed at positions $\mp x_{0}$. The Hamiltonian for electrons 1 and 2 becomes

$$
\begin{gathered}
H=\underbrace{T_{1} V_{1 a}}_{=H_{1}+H_{2}+V_{12}}+V_{1 b}+\underbrace{T_{2}+V_{2 b}}_{1 a}+V_{2 a}+V_{12} \\
H_{2 b}+V_{2 a} \\
H_{12}
\end{gathered}
$$

where

$$
\begin{array}{cc}
T_{1}=-\left(\frac{\hbar^{2}}{2 m}\right) \frac{\partial^{2}}{\partial x_{1}^{2},} & T_{2}=-\left(\frac{\hbar^{2}}{2 m}\right) \frac{\partial^{2}}{\partial x_{2}^{2}}, \\
V_{1 a}=-A \delta\left(x_{1}+x_{0}\right), & V_{2 a}=-A \delta\left(x_{2}+x_{0}\right), \\
V_{1 b}=-A \delta\left(x_{1}-x_{0}\right), & V_{2 b}=-A \delta\left(x_{2}-x_{0}\right), \\
V_{12}=A \delta\left(x_{1}-x_{2}\right) .
\end{array}
$$

The grouping of terms in (3a) and (3b) will be useful in the following considerations. The electron-electron repulsion $V_{12}$ is, as other Coulomb interactions, simplified to a $\delta$ function. In this case the sign is positive, and the argument is $x_{1}-x_{2}$. This term makes impossible the complete separation into 2 one-particle Hamiltonians, which would have made the problem trivial.

Hence, the true eigenfunctions for the two-electron Hamiltonian (3) are not readily obtainable, and we are, as for the $\mathrm{H}_{2}$ problem, left with the trial methods. We must guess a reasonable ground-state wave function $u\left(x_{1}, x_{2}\right)$ and evaluate $E=\langle H\rangle$, which will be an upper limit for the true ground-state energy.

\section{FIRST TRIAL: ATOMIC ORBITAL PRODUCT (AO)}

The simplest conceivable test function seems to be

$$
u\left(x_{1}, x_{2}\right)=a\left(x_{1}\right) b\left(x_{2}\right),
$$

where $a$ and $b$ are "atomic orbitals" (2) associated with each of the two nuclei

$$
a(x) \equiv v\left(x+x_{0}\right), \quad b(x) \equiv v\left(x-x_{0}\right) .
$$

For the calculation of $E$, the Hamiltonian is used in the form (3a). The test function (4) is normalized and real, and is a simultaneous eigenfunction for $H_{1 a}$ and $H_{2 b}$ with the eigenvalue $-E_{0}$. The electron-electron term $V_{12}$ yields a 
positive contribution $j$, and $V_{1 b}$ and $V_{2 a}$ give rise to equal and negative contributions $-j^{\prime}$, where

$$
\begin{aligned}
j & \equiv \iint a\left(x_{1}\right)^{2} A \delta\left(x_{1}-x_{2}\right) b\left(x_{2}\right)^{2} d x_{1} d x_{2} \\
& =E_{0}\left(1+4 \beta x_{0}\right) \exp \left(-4 \beta x_{0}\right), \\
j^{\prime} & \equiv \int a\left(x_{1}\right)^{2} A \delta\left(x_{1}-x_{0}\right) d x_{1} \\
& =\int b\left(x_{2}\right)^{2} A \delta\left(x_{2}+x_{0}\right) d x_{2}=2 E_{0} \exp \left(-4 \beta x_{0}\right) .
\end{aligned}
$$

It is the intended merit of our crude model that these "Coulomb integrals" (and other integrals to come) are easy to calculate, and that the results are simple analytical functions of the interatomic distance $2 x_{0}$. The first integral (6) just involves one-dimensional integration of a pieceby-piece exponential function, whereas Eq. (7) comes out merely an evaluation of an exponential at a single point.

The total electronic energy of the simple trial function (4) becomes

$$
\begin{aligned}
E=-2 E_{0}+ & j-2 j^{\prime} \\
& =E_{0}\left[-2+\left(-3+4 \beta x_{0}\right) \exp \left(-4 \beta x_{0}\right)\right]
\end{aligned}
$$

which is shown in Fig. 1 as the curve labeled AO. The asymptotic value for large interatomic distances is $-2 E_{0}$, corresponding to two atomic binding energies. A weak barrier appears around $2 \beta x_{0}=2$, while the limiting value for $x_{0} \rightarrow 0$ is $-5 E_{0}$ (compared to $-4.75 \mathrm{Ry}$ for the $\mathrm{H}_{2}$ problem ${ }^{3}$ ).
Coulomb repulsion between nuclei has not been included in our considerations, since a $\delta$-potential approximation would not be appropriate in this place (heavy particle motion, classical treatment). Therefore, numbers for molecular binding energy and equilibrium interatomic distance cannot be derived, but this will not affect the comparison of different trials on the basis of electronic energy $E\left(x_{0}\right)$ alone.

\section{SECOND TRIAL: SYMMETRIZED ATOMIC ORBITAL FUNCTIONS (AOS,AOT)}

An obvious defect of the first trial function (4) is that it does not satisfy the Pauli principle. In analogy with the Heitler-London procedure for the $\mathrm{H}_{2}$ problem one should assume spatial functions that are symmetric or antisymmetric with respect to particle permutation:

$$
u\left(x_{1}, x_{2}\right)=a\left(x_{1}\right) b\left(x_{2}\right) \pm a\left(x_{2}\right) b\left(x_{1}\right)
$$

and combine the + function with the antisymmetric twospin function (singlet, AOS) or the - function with one out of three possible symmetric two-spin functions (triplet, AOT).

Calculation of electronic energy is again performed with the Hamiltonian in the form ( $3 a$ ) and by exploiting the symmetry of $H$ with respect to particle permutation. The test functions (9) are real but not normalized, so that

$$
\begin{aligned}
E & =\left[\iint u\left(x_{1}, x_{2}\right) H u\left(x_{1}, x_{2}\right) d x_{1} d x_{2}\right] /\left[\iint u\left(x_{1}, x_{2}\right)^{2} d x_{1} d x_{2}\right] \\
& =\frac{\iint a\left(x_{1}\right) b\left(x_{2}\right) H a\left(x_{1}\right) b\left(x_{2}\right) d x_{1} d x_{2} \pm \iint a\left(x_{2}\right) b\left(x_{1}\right) H a\left(x_{1}\right) b\left(x_{2}\right) d x_{1} d x_{2}}{\iint a\left(x_{1}\right)^{2} b\left(x_{2}\right)^{2} d x_{1} d x_{2} \pm \iint a\left(x_{1}\right) b\left(x_{1}\right) a\left(x_{2}\right) b\left(x_{2}\right) d x_{1} d x_{2}} \\
& =\left[\left(-2 E_{0}+j-2 j^{\prime}\right) \pm\left(-2 S^{2} E_{0}+k-2 S k^{\prime}\right)\right] /\left(1 \pm S^{2}\right) \\
& =-2 E_{0}+\left[\left(j-2 j^{\prime}\right) \pm\left(k-2 S k^{\prime}\right)\right] /\left(1 \pm S^{2}\right) .
\end{aligned}
$$

Here we have introduced the overlap integral $S$ and the exchange integrals $k$ (overlap-overlap) and $k^{\prime}$ (overlapnucleus), which are all very easily calculated:

$$
\begin{aligned}
& S \equiv \int a(x) b(x) d x=\left(1+2 \beta x_{0}\right) \exp \left(-2 \beta x_{0}\right), \\
& k \equiv \iint a\left(x_{1}\right) b\left(x_{1}\right) A \delta\left(x_{1}-x_{2}\right) a\left(x_{2}\right) b\left(x_{2}\right) \\
& \times \times x_{1} d x_{2}=E_{0}\left(1+4 \beta x_{0}\right) \exp \left(-4 \beta x_{0}\right), \\
& k^{\prime} \equiv \int a\left(x_{1}\right) b\left(x_{1}\right) A \delta\left(x_{1}-x_{0}\right) d x_{1} \\
&= \int a\left(x_{2}\right) b\left(x_{2}\right) A \delta\left(x_{2}+x_{0}\right) d x_{2} \\
&= 2 E_{0} \exp \left(-2 \beta x_{0}\right) .
\end{aligned}
$$

The curves AOS (singlet, plus sign) and AOT (triplet, minus sign) in Fig. 1 show the electronic energies Eq. (10) for the trial functions Eq. (9). The triplet state energy diverges for $x_{0} \rightarrow 0$, whereas the singlet state gives rise to a stronger interatomic attraction than the primitive product function Eq. (4) and is therefore, so far, the best approximation to the two-electron ground state. The improvement is immediately traced to be connected with the exchange term containing $k^{\prime}$. The electron density distribution $\rho(x)$ can very easily be calculated for the investigated trial functions. So it can be demonstrated that the stronger binding is associated with an enhanced electron density between the nuclei $\left(-x_{0}<x<x_{0}\right)$.

\section{INSERTION ABOUT A ONE-ELECTRON PROBLEM: THE $\delta_{2}^{+}$ION}

The Schrödinger equation for a single electron in the one-dimensional potential set up by the two $\delta$ nuclei is easily solved. Exact solutions can be found just as for the $\delta$ atom, but it will appear more instructive to consider the approximate solutions found by linear combination of the atomic orbitals $a$ and $b$ Eq. (5) (LCAO method). Due to spatial symmetry the two proper combinations must be even and odd functions of $x$. Normalization and calculation of energies as averages of the one-electron Hamiltonian only involve already treated integrals, and the results are

$$
\begin{array}{ll}
B(x)=\frac{a(x)+b(x)}{[2(1+S)]^{1 / 2}}, & E_{B}=-\left(E_{0}+\frac{j^{\prime}+k^{\prime}}{1+S}\right) \\
A(x)=\frac{a(x)-b(x)}{[2(1-S)]^{1 / 2}}, & E_{A}=-\left(E_{0}+\frac{j^{\prime}-k^{\prime}}{1-S}\right) .
\end{array}
$$

$B$ is a bonding orbital with an energy below $-E_{0}$ and a high electron density between the nuclei. $x_{0}=0$ leads to $E_{B}=$ $-3 E_{0}$, corresponding to $-3 \mathrm{Ry}$ for the LCAO treatment of $\mathrm{H}_{2}^{+} . A$ is an antibonding orbital with energy higher than $-E_{0}\left(E_{A} \rightarrow \infty\right.$ for $\left.x_{0} \rightarrow 0\right)$ and zero electron density for $x$ $=0$.

\section{THIRD TRIAL: MOLECULAR ORBITAL METHOD (MO)}

The molecular orbital method can now be applied to the two-electron problem of the $\delta_{2}$ molecule. Two electrons (opposite spins) are filled into the bonding orbital, and the following normalized trial function (singlet) is obtained: 


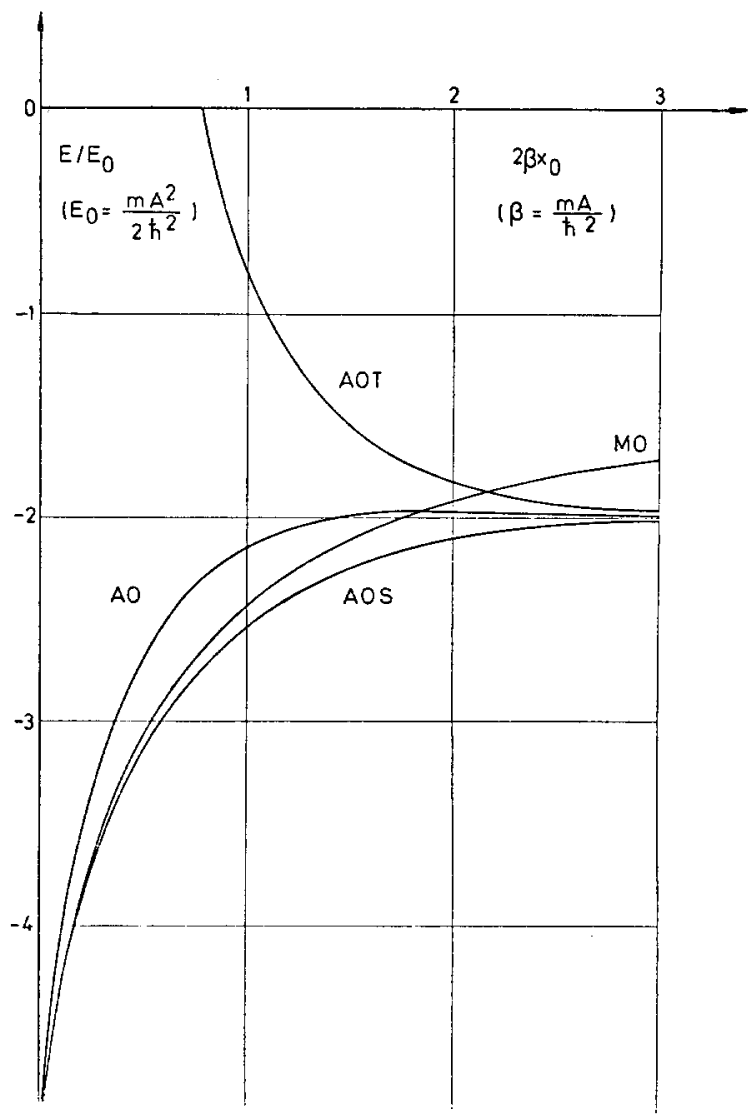

Fig. 1. Electronic energy vs interatomic distance for different trial functions. AO, atomic orbital product; AOS, AOT, symmetrized atomic orbital functions (singlet, triplet); MO, molecular orbital product.

$$
\begin{aligned}
u\left(x_{1}, x_{2}\right)= & B\left(x_{1}\right) B\left(x_{2}\right) \\
& =\left[a\left(x_{1}\right) a\left(x_{2}\right)+b\left(x_{1}\right) b\left(x_{2}\right)\right. \\
& \left.+a\left(x_{1}\right) b\left(x_{2}\right)+b\left(x_{1}\right) a\left(x_{2}\right)\right] / 2(1+S) .
\end{aligned}
$$

The energy is obtained by using the Hamiltonian in the form (3b), where $H_{1}$ and $H_{2}$ give rise to equal contributions $E_{B}[\mathrm{Eq} .(14)]$, and the electron-electron repulsion yields $J /(1+S)^{2}$ with

$$
\begin{gathered}
J \equiv \frac{1}{4} \iint\left[a\left(x_{1}\right)+b\left(x_{1}\right)\right]^{2} A \delta\left(x_{1}-x_{2}\right) \\
\times\left[a\left(x_{2}\right)+b\left(x_{2}\right)\right]^{2} d x_{1} d x_{2}=E_{0}\left[1 / 2+3 \exp \left(-2 \beta x_{0}\right)\right. \\
+\left(3 / 2+6 \beta x_{0}\right) \exp \left(-4 \beta x_{0}\right) \\
\left.-\exp \left(-6 \beta x_{0}\right)\right] .
\end{gathered}
$$

This integral involves a combination of exponential functions but is still straightforward to handle. The total electronic energy,

$$
E=-2 E_{0}-2\left(j^{\prime}+k^{\prime}\right) /(1+S)+J /(1+S)^{2}
$$

is shown in Fig. 1 with the curve labeled MO. For small interatomic distances the result is seen to be very close to that obtained for the AOS function (the difference is of second order in $\beta x_{0}$ ). An even stronger binding could have been demonstrated by using the exact molecular orbitals. As far as concerns the electron density distribution $\rho(x)$ the $\mathrm{MO}$ and AOS approximations are very similar, but the correlation between the two electrons is entirely different in the two cases. Thus the MO wave function Eq. (15) contains terms of the type $a\left(x_{1}\right) a\left(x_{2}\right)$ or $b\left(x_{1}\right) b\left(x_{2}\right)$, which describe situations with both electrons centered around the same nucleus. For $x_{0} \rightarrow \infty$ this leads to a too high electron energy $\left(-1.5 E_{0}\right)$, because the molecule can dissociate into $\delta^{+}$and $\delta^{-}$ions instead of two neutral $\delta$ atoms.

\section{ALTERNATIVE INTERPRETATION OF THE TWO-PARTICLE HAMILTONIAN}

The Hamiltonian Eq. (3) might alternatively be looked at as an operator for one particle with two degrees of freedom $\left(x_{1}, x_{2}\right)$. The two-dimensional potential function for this particle is zero everywhere, except for sharp ditches along the lines $x_{1}= \pm x_{0}$ and $x_{2}= \pm x_{0}$, and a sharp barrier along $x_{2}=x_{1}$.

The previous discussion of particle permutation $\left(x_{1} \leftrightarrow\right.$ $x_{2}$ ) is now turned to a discussion of a spatial symmetry, as $x_{2}=x_{1}$ is a mirror line for the potential energy function. The alternative interpretation opens no new ways of finding exact solutions to the wave equation, but it offers the possibility of making instructive pictures of the potential energy and the wave functions as shown in Fig. 2.

\section{VARIATION METHODS}

The simplicity of the molecule model presented here makes it well suited for further exercises, such as improvements of trial functions by means of variation methods.

Let us consider as an example the special case $x_{0}=0$, which is analogous to a $\mathrm{He}$ atom rather than a $\mathrm{H}_{2}$ molecule. The Hamiltonian Eq. (3) reduces to

$$
\begin{aligned}
& H=-\left(\hbar^{2} / 2 m\right)\left(\partial^{2} / \partial x_{1}^{2}+\partial^{2} / \partial x_{2}^{2}\right) \\
& -Z A\left[\delta\left(x_{1}\right)+\delta\left(x_{2}\right)\right]+\lambda A \delta\left(x_{1}-x_{2}\right)
\end{aligned}
$$

with $Z=2$ and $\lambda=1$. A natural choice of parametrized trial function will be

$$
u\left(x_{1}, x_{2}\right)=\beta \exp \left[-\beta\left(\left|x_{1}\right|+\left|x_{2}\right|\right)\right], \quad \beta=b m A / h^{2} .
$$

When the variation parameter $b$ equals unity, this is the
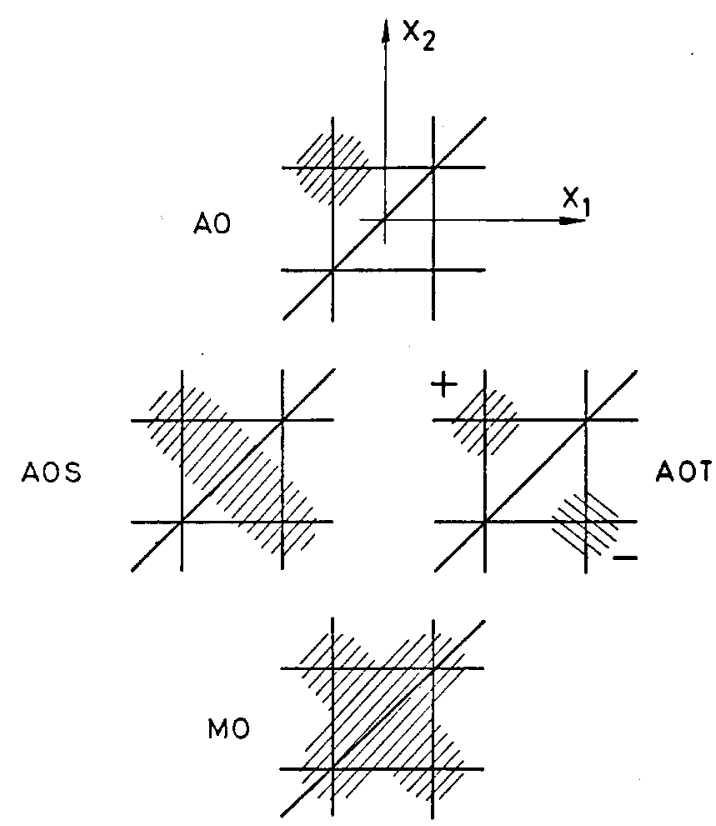

Fig. 2. Schematic illustrations of potential energy function (ditches and barrier) and wave functions (shaded regions). 
normalized limiting function for the previous trial functions Eqs. (4), the plus function of (9), and (15) as $x_{0} \rightarrow 0$. Calculation of energy is easily carried out, when attention is paid to the discontinuities of the wave-function derivatives at $x_{1}=0$ and $x_{2}=0$ ( $\delta$ contributions to the kinetic energy). The result turns out to be

$$
\begin{aligned}
E=\hbar^{2} \beta^{2} / m-A \beta(2 Z-\lambda / 2) & =-E_{0}\left[(8-\lambda) b-2 b^{2}\right] .
\end{aligned}
$$

The electron-electron repulsion strength has been left as a parameter $\lambda$, so that the following four cases can be considered:

(i) $\lambda=1, b=1: E=-5 E_{0}$ as previously found.

(ii) $\lambda=1$ : The energy Eq. (20) has a minimum $E$ $=-49 / 8 E_{0}=-6.125 E_{0}$ for $b=7 / 4=1.75$. The corresponding values of the $\mathrm{He}$ atom are $-5.7 \mathrm{Ry}$ for an effective nuclear charge $b=27 / 16 \cong 1.69$.

(iii) $\lambda=0$ : Without electron-electron repulsion Eq. (19) is an exact solution for $b=2$. Equation (20) yields $E$ $=-8 E_{0}$. Thus the correct energy must be found in the interval between $-8 E_{0}$ and $-6.125 E_{0}$.

(iv) If the repulsion term is treated as a perturbation, Eq. (20) with $b=2$ gives the energy to first order in $\lambda . \lambda=1$ yields $E=-6 E_{0}$.

\section{CONCLUSION}

The proposed simplified model of a diatomic molecule has been shown to display all the essential features of a real two-particle quantum-mechanical system, and furthermore to yield results in semiquantitative agreement with those for the $\mathrm{H}_{2}$ molecule. Nevertheless, the practical calculation of integrals is extremely simple and therefore allows introduction of the model as an instructive student exercise.

'See, for instance, J. C. Slater, Quantum Theory of Molecules and Solids (McGraw-Hill, New York, 1963), Vol. 1, "Electronic structure of Molecules."

${ }^{2}$ The boundary condition for $x=0$ is obtained by integration of Eq. (1) from $-\epsilon$ to $\epsilon$. For $\epsilon \rightarrow 0$ this yieids

$$
v^{\prime}\left(0^{+}\right)-v^{\prime}\left(0^{-}\right)=-\left(2 m A / h^{2}\right) v(0) .
$$

See also D. A. Atkinson and H. W. Crater, Am. J. Phys. 43, 301 (1975).

${ }^{3}$ This is the result of the Heitler-London method (Ref. 1, Fig. 3-3), which corresponds rather to our second trial (AOS); but the two approaches are in fact identical in the limiting case $x_{0}=0$. 